\title{
Lacrimal canaliculitis- A case report
}

\author{
Dr. L. Usharani ${ }^{1}$, Dr. Gaining Lulu Kamei ${ }^{2}$, Dr. Y. Chingsuingamba Meitei ${ }^{3}$ \\ 1,2,3 (Department of Ophthalmology, Regional Institute of Medical Sciences,Imphal.Manipur)
}

\begin{abstract}
Lacrimal canaliculitis is an infection of the lacrimal duct system. It is an uncommon, often misdiagnosed diagnosis because it can mimic many other common ocular conditions. The classic features of lacrimal canaliculitis are mild to severe swelling of the canaliculus, mucopurulent discharge from the punctum, and a red, pouting punctum.Canaliculitis should be appropriately diagnosed and treated to avoid recurrent inflammation and possible obstruction of the upper portion of the lacrimal system.However, Primary chronic canaliculitis may account for approximately $2 \%$ of all tearing problems. Actinomycosis may form in up to $2 \%$ of all lacrimal disease and can cause conjunctivitis or lacrimal canaliculitis with no generalized systemic invasion.Canaliculotomy with systemic or topical antibiotics is the most appropriate treatment for this disorder. We report a case of lacrimal canaliculitis in a 57years old female who presented in our opd with painless swelling of the right medial upper lid and occasional redness for 2 years. Despite surgical management being the definitive treatment, the patient was managed conservatively with no recurrence till date.
\end{abstract}

Key words: Actinomyces israelii, canaliculotomy, Lacrimal canaliculitis, punctum.

\section{Introduction}

Canaliculitis is an inflammation of the lacrimal canaliculi caused by infection ${ }^{1}$ or as a complication of punctal plug insertion. ${ }^{2,3}$ It is often misdiagnosed ${ }^{4}$ leading to a delay in diagnosis. ${ }^{5}$ Canaliculitis is generally a unilateral condition. ${ }^{5}$ Symptoms associated with primary and secondary canaliculitis include epiphora, conjunctivitis, eyelid matting, a swollen, pouting punctum, or purulent discharge. ${ }^{4,6,7}$ Other findings include sulfur granules or canaliculiths extruded from the punctum via massage or discovered during canaliculotomy. ${ }^{6,8}$ Additional findings with cases of secondary canaliculitis include an inflammatory mass projecting from the punctum or intermittent blood-stained tears. ${ }^{2,3,8}$ Canaliculitis should be considered as a possible etiology with persistent or recurrent eyelid lesions, chalazia, or abscesses. ${ }^{9}$ A primary infection of the lacrimal canaliculus is relatively uncommon. Actinomycosis may form in up to $2 \%$ of all lacrimal disease. ${ }^{10}$

\section{Case History}

A 57 years old female patient presented in the eye opd with complaints of swelling of the medial $1 / 4$ of the right upper lid since two years with redness off and on.The swelling was painless and gradually increasing in size.She also gives history of whitish granular discharge from the upper punctum about one year after the appearance of the swelling.There was no history of injury locally. Family history and personal history were not significant.

\section{Ophthalmological examination}

The best corrected visual acuity of both eyes was 6/6 Plano,N6 with +1.50DS.

Right eye showed bulbar conjunctival congestion nasally and non tender swelling in the upper canalicular region with pouting of the upper punctum(fig. 1).

Syringing was patent in the right lower canaliculus but the upper right canaliculus could not be cannulated. Fundus and rest of the ocular examination of both eye was within normal limit.

\section{Management}

Initially,the patient was started on broad spectrum systemic as well as topical antibiotic . She did not respond to the treatment. Later she was taken to the minor operation theatre where intraoperatively large amount of granular cheesy sticky discharge was expressed from the upper punctum on gentle pressure.The discharge material was sent for microbiological examination. A diagnosis of right upper canaliculitis was made based on the clinical and Ophthalmological findings.

The report showed Actinomyces israelii which was sensitive to levofloxacin,piperacillin,tazobactam, gentamycin,azithromycin,ofloxacin and ciprofloxacin but resistant to cefadroxil.

She was started on on azithromycin systematically along with topical moxifloxacin eyedrop and gentamycin eye ointment.On the first day of follow up there was mucoid discharge and mild swelling but by the $4^{\text {th }}$ day the swelling along with the discharge subsided and ,since then, on subsequent follow up visits the patient has not been having any complaint(fig.2). 


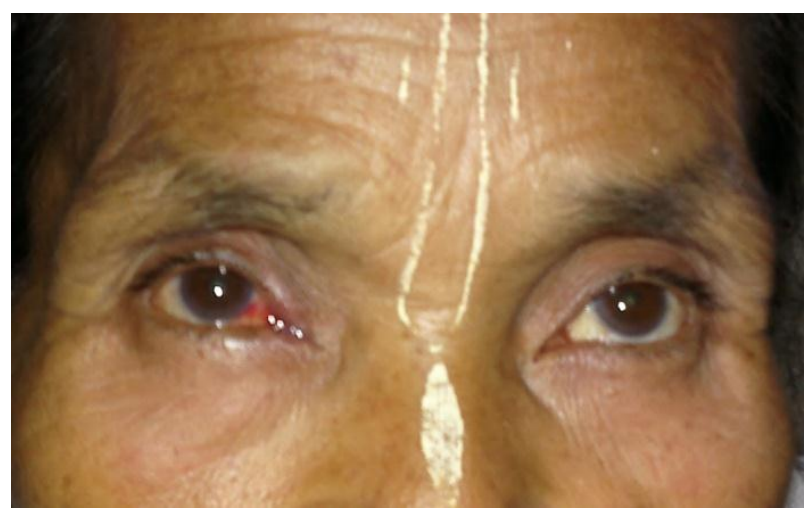

Fig.1 Lacrimal canaliculitis of right eye with conjunctival injection nasally

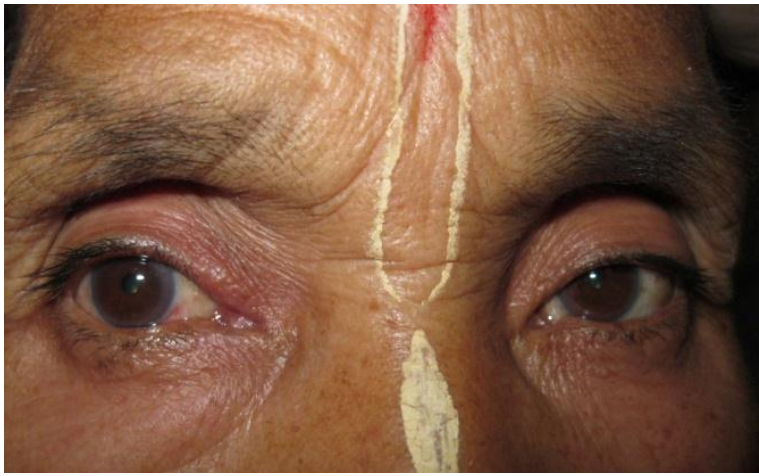

Fig.2 Showing post treatment

\section{Discussion}

Lacrimal canaliculitis results from infection of the canaliculi and is most frequently caused by Actinomyces Israelii,an anaerobic gram positive fungus which aggregates to produce large cheesy masses within the distended canal. ${ }^{11}$ Actinomyces is difficult to culture and microbiological diagnosis may be complicated by secondary bacterial infection such as Staphylococcus. ${ }^{12}$ The cheesy secretion may block the flow of tears and yet,the canaliculus often remain patent to syringing. Pressure over the canaliculus expresses a thick discharge from the punctum.Lacrimal canaliculitis has been reportedly misdiagnosed as mucoceles,dacryocystitis,blepharitis and chalazia. ${ }^{13}$ Lacrimal canaliculitis can often be a cause of non resolving conjunctivitis and is often misdiagnosed. ${ }^{14}$ Therefore it is important to keep in mind this condition while seeing any of the above mentioned cases. The surgical treatment of canaliculitis in combination with conservative therapy yields far better results than conservative therapy alone. ${ }^{15}$ Definitive cure alone will not be affected until all concretion are removed,either at surgery or by mechanical expression ${ }^{2}$ and subsequent treatment with topical and systemic antibiotic.

\section{Conclusion}

Epiphora, chronic or recurrent unilateral conjunctivitis, pouting punctum, yellow discharge or canaliculiths are all presentations of canaliculitis. If discharge or concretions are present, microbiological or histopathological investigation should be conducted from expressed materials. First line treatment is conservative medical therapy with more invasive therapy reserved for conservative treatment failure. It is important to consider the diagnosis of lacrimal canaliculitis in any patient with chronic or recurrent conjunctivitis. Greater awareness of this condition would prevent misdiagnosis and delays in referral for definitive treatment.

\section{References}

[1]. Baldursdottir E, Sigurdsson H, Jonasson L, Gottfredsson M. Actinomycotic canaliculitis: resolution following surgery and short topical antibiotic treatment. Acta Ophthalmol 2010May;88(3):367-70.

[2]. Smartplug study group.Management of complications after insertion of the SmartPlug punctal plug: a study of 28 patients. Ophthalmology 2006Oct;113(10):1859.

[3]. Scheepers M, Pearson A, Michaelides M. Bilateral canaliculitisfollowing SmartPlug insertion for dry eye syndrome post LASIK surgery. Graefes Arch Clin Exp Ophthalmol 2007;245:895-7.

[4]. Fulmer NL, Neal JG, Bussard GM,Edlich RF. Lacrimal canaliculitis. Am J Emerg Med 1999July;17(4):385-6.

[5]. Kaliki S, Ali MJ, Honavar SG, Chandrasekhar G,Naik MN. Primary canaliculitis: clinical features, microbiological profile, and management outcome. Ophthal Plast Reconstr Surg 2012;28:355-60. 
[6]. Demant E, Hurwitz JJ. Canaliculitis: review of 12 cases. Can JOphthalmol 1980;15:73-5.

[7]. Zaldivar RA, Bradley EA. Primary canaliculitis. Ophthal Plast Reconstr Surg 2009;25:481-4.

[8]. Park A, Morgenstern KE, Kahwash SB,Foster JA. Pediatric canaliculitis and stone formation. Ophthal Plast Reconstr Surg 2004;20:243-6.

[9]. Almaliotis D, Nakos E, Siempis T,Koletsa T,Kostopoulos I,Chatzipantazi M, Karampatakis V. A para-canalicular abscess resembling an inflamed chalazion. Case Rep Ophthalmol Med 2013;2013.

[10]. Takemura M, Yokoi N, Nakamura Y, Komuro A, Sugita J, Kinoshita S. Canaliculitis caused by Actinomyces in a case of dry eye punctual plug occlusion. Nihon Ganka Gakkai Zasshi 2002 Jul;106(7):416-9.

[11]. PD Trevor-Roper,PV Curran.Disorder of the lacrimal apparatus.In: PD Trevor-Roper,PV Curran , editors.The Eye and its disorders. $2^{\text {nd }}$ edition.Blackwell Scientific Publications.Oxford; 1984;303-304.

[12]. Varma D, Chang B,Musaad S.A case series on chronic canaliculitis.Orbit 2005;24(1):11-14.

[13]. Anand S, Hollingworth K, Kumar V,Sandramouli S.Canaliculitis:the incidence of long -term epiphora following canaliculotomy.Orbit.2003;23(1):19-26.

[14]. Sidath E.Liyanage,Michael W.Lacrimal canaliculitis as a cause of recurrent conjunctivitis.Journal of American Optometric Association.2009;80(9):479-480.

[15]. Vecsei VP,Huber-Spitzy V,Arocker-Mettinger E, Steinkogler FJ.Canaliculitis:difficulties in diagnosis,differential diagnosis and comparasion between conservative and surgical treatment.Ophthalmologica.1994;208(6):314-317. 